\title{
Association of health literacy and medication adherence with health-related quality of life (HRQoL) in patients with ischemic heart disease
}

\author{
Muzna Suhail ${ }^{1}$, Hamid Saeed ${ }^{1 *}\left(\mathbb{D}\right.$, Zikria Saleem²$^{2}$, Saman Younas ${ }^{1}$, Furqan Khurshid Hashmi ${ }^{1}$, Fawad Rasool ${ }^{3}$, \\ Muhammad Islam ${ }^{1}$ and Imran Imran ${ }^{4}$
}

\begin{abstract}
Background: Medication Adherence (MA) and Health Related Quality of Life (HRQoL) are two inter-connected concepts, co-influenced by Health Literacy $(\mathrm{HL})$, with significant impact on patient management and care. Thus, we aimed to estimate the association of $\mathrm{HL}$ and MA with HRQoL in IHD patients.

Methods: Cross-sectional study of 251 IHD patients recruited from Lahore over 6 months period. HL, MA and HRQoL was assessed using validated questionnaires; 16-items of HL, Morisky Green Levine Scale (MGLS) and SF-12, respectively. Chi-square for significance, logistic-regression for association and linear regression for predictions were used.

Results: IHD patients; males ( $p=0.0001)$, having secondary—higher education ( $p=0.0001)$, middle/upper class $(p=0.0001)$ and employed $(p=0.005)$ had adequate $\mathrm{HL}$, and were more likely to be adherent $(\mathrm{OR} ; 4.3, p=0.014)$. Both physical (PCS-12) and mental (MCS-12) component scores of HRQoL for age, gender, education, area of residence, employment and MA were significantly higher in patients with adequate $\mathrm{HL}$. In multinomial regression, improved PCS-12 scores tend to be higher in subjects having secondary —higher education (OR; 3.5, $p=0.067$ ), employed (OR; $6.1, p=0.002)$ and adherent (OR; $2.95, p=0.218)$, while MCS-12 scores tend to be higher in patients $<65$ years (OR; 2.2 , $p=0.032)$, employed (OR; $3, p=0.002)$ and adherent (OR; $4, p=0.004)$. In adjusted model, $\mathrm{HL}(\beta ; 0.383, p=0.0001)$ and MA $(\beta ;-0.133, p=0.018)$ were significantly associated with PCS-12, and MCS-12 with MA $(\beta ;-0.161, p=0.009)$ only.
\end{abstract}

Conclusion: Data suggested that adequate HL was significantly associated with adherence and both physical and mental dimensions of HRQoL were higher in IHD patients with adequate HL. Besides, HL and MA are independent predictors of HRQOL in IHD patients.

Keywords: Medication adherence, Health literacy, HRQoL, Ischemic heart disease, Lahore, Pakistan

\section{Introduction}

Ischemic health disease (IHD), the most common cardiovascular disease (CVD), is the leading cause of morbidity and mortality worldwide [1]. According to one estimate almost 17.5 million people died from CVDs in 2012, representing $31 \%$ of all global deaths [2]. Surprisingly, over

\footnotetext{
*Correspondence: hamid.pharmacy@pu.edu.pk

${ }^{1}$ Department of Pharmaceutics, University College of Pharmacy, Universality of the Punjab, Allama labal Campus, Lahore 54000, Pakistan

Full list of author information is available at the end of the article
}

three quarters of all deaths due to CVD took place in low and middle-income countries [3-5]. Due to complex nature of the disease, the contemporary management of IHD require multifaceted approach-aimed not only on reducing the morbidity and mortality but also improving the quality of life $[6,7]$.

Health literacy (HL), a cognitive and social skill that enable individuals to access, understand and utilized health information to preserve good health, is a potential risk factor for health outcomes related to physical original author(s) and the source, provide a link to the Creative Commons licence, and indicate if changes were made. The images or other third party material in this article are included in the article's Creative Commons licence, unless indicated otherwise in a credit line to the material. If material is not included in the article's Creative Commons licence and your intended use is not permitted by statutory regulation or exceeds the permitted use, you will need to obtain permission directly from the copyright holder. To view a copy of this licence, visit http://creativecommons.org/licenses/by/4.0/. The Creative Commons Public Domain Dedication waiver (http://creativeco mmons.org/publicdomain/zero/1.0/) applies to the data made available in this article, unless otherwise stated in a credit line to the data. 
functioning, such as poor medication adherence, selfmanagement of the disease, health behaviors and reduced utilization of preventive services [8]. Thus, sufficient understanding of health information, disease condition, medication and commitment to prolong therapy is pivotal for IHD patients, and due to chronic nature of the disease they are required to actively participate in the management of their health condition $[9,10]$. Nevertheless, for chronic disease management, medications are always the foremost aces, yet, adherence to long-term therapy is persistently poor in such patients [11], which may have severe health related consequences especially for patients with cardiovascular diseases [12]. On an average, for the chronic disease management, almost $50 \%$ patients fail to adhere to disease preventive measures, such as taking medication, keeping appointments, screening, exercise and dietary changes [13, 14], which is mainly driven by lack of understanding about the disease and associated medication therapy $[15,16]$

Medication adherence (MA), the extent to which a person's behavior, taking medication, following a diet and life style changes corresponds with agreed recommendations from a health care provider, has been shown to be higher among patients with acute illness compared to those having chronic illness $[17,18]$. Several lines of evidences suggest that adherence to cardiovascular medication is sub-optimal in patients with hypertension, coronary heart disease and ischemic heart disease, which continues to undermine their therapeutic efficacy-leading to poor outcomes and higher costs for patients and the health care system $[19,20]$

More recently, HRQoL has become an important endpoint in the evaluation of health interventions, particularly in patients with chronic health conditions [21]. HRQoL is defined as the extent to which one's expected physical, social and emotional well-being is affected by his/her medical condition or the treatment [22]. Literature evidences suggest that both health literacy (HL) and medication adherence (MA), alone or in support, decisively affects health related quality of life (HRQoL) in patient with chronic diseases, such as coronary artery disease, hypertension and diabetes [14, 23-27]. Contrariwise, inadequate health literacy and medication non-adherence can adversely affect the cost of therapy, health outcomes and HRQoL, especially in patients with chronic diseases [23, 27-31]. Several patient related factors affecting patient's outcomes, such as disease and medication knowledge, lifestyle and behavioral changes, affordability and active self-management of their health conditions, all gambling on HL associated MA, have been shown to impact the HRQoL [24, 32-34]. Seemingly, optimal HL and adherence to medication regimen are critical in improving HRQoL, especially where clinicians struggle to achieve the primary and secondary treatment targets [35].

In Pakistan, where adult literacy stands at 55\% (men $67 \%$ and women $42 \%),[14,36,37]$ and majority of the patients with chronic diseases are elderly, having low income, poor education and carry the highest disease burden that may adversely affect the self-care practices, health care usage and disease management [38]. The HRQoL among IHD patients is influenced by several objective and subjective factors that may act together. Keeping in view the above-mentioned existing literature reports, HL, MA and HRQoL may be related and have intricate yet complex relationships that require further investigations-particularly in Pakistani IHD population having poor understanding of the disease and about the treatments. Among South Asian countries, Pakistan has the lowest literacy rate [39] and significantly higher IHD disease burden, though very few literature evidences are available from Pakistan that deal with the impact of HL on HRQoL in patients with ischemic heart disease (IHD) [24], however, not a single study examined the impact of both, HL and MA, significantly associated with each other, on the HRQoL in IHD patients. Thus, the present study was conducted with three purposeful objectives, first the impact of HL on physical and mental components scores of HRQoL, second to estimate the association of HL and HRQoL with patient's demographics, clinical characteristics and MA and lastly to determine the predictors of physical and mental HRQoL based on HL and MA scores in IHD patients.

\section{Methodology \\ Ethical approval}

The present study was approved from the Human Ethical Committee University College of Pharmacy, University of the Punjab, Lahore, reference number HEC/PUCP/1930, and from Institutional Review Board (IRB), King Edward Medical University, Lahore, reference number 320/ $\mathrm{RC} / \mathrm{KEMU}$. Informed consent was obtained from the participants.

\section{Study design}

A descriptive cross-sectional study was designed to investigate the impact of HL and MA on HRQoL in IHD patients. The study consisted of three distinct portions, i.e. (a) Evaluation of health literacy using a validated 16 items questionnaire developed by chew et al. [40], (b) assessment of MA using Morisky Green Levine Medication Adherence Scale (MGLS) and (c) the impact of both on health-related quality of life (HRQOL) evaluated employing 12-Item Short Form Survey, (SF-12) version 1.0. 


\section{Study settings}

Patients were recruited from one of the biggest tertiary care hospital of Lahore, i-e., Mayo hospital ( $\mathrm{MH})$ [41], between June 2016-Nov 2016, with influx of patients from all over the Punjab province having estimated population of 110 million [42]. Mayo Hospital was established in 1871 with 2399 beds capacity located in Lahore encomapssing 54.6 acers of land. MH provides, clinical (in-patient \& out-patient), diagnostic and emergency services [41]. IHD patients were enrolled from hospital's out-patient cardiology department.

A comprehensive instrument of measure (questionnaire) was used to collect the data. Questionnaire was administered by field administrator, post graduate pharmacy student, by filling the questionnaire during face-toface interviews.

\section{Participants}

A total of 251 eligible IHD patients with confirmed clinical diagnosis, as per American College of Cardiology guidelines [43] by a cardiologist, were enrolled in the study from out-patient cardiology department as per study inclusion and exclusion criteria.

Inclusion criteria All the eligible IHD patients having confirmed diagnosis as per cardiologist report, above 25 years of age, with disease duration of more than 6 months, irrespective of gender, socio-economic status, ethnicity, the presence of co-morbid conditions and willing to provide informed consent were included in the study.

Exclusion criteria All those patients below 25 years of age, disease duration of less than 6 months, terminally ill, cognitive impairment and failed to provide the informed consent were excluded.

Randomization was ensured by giving serial numbers $(1,2,3 \ldots)$ to all the eligible patients as per clinician's confirmed IHD diagnosis and later segregating even numbered patients into the study group.

\section{Variables and data measure}

Study variables are categorized into independent and dependent (outcome) variables.

\section{Independent variable}

Health Literacy was considered an independent variable and was evaluated using a validated 16 item questionnaire developed by chew et al. [40]. Health literacy tool (16-items); a valid questionnaire developed by chew et al. in which every question was rated on a 5-point scale ranging from 0 (always) to 4 (never) except for questions $1-4,14$ and 15 which were reverse coded for calculating health literacy levels, the score ranged from 0-64, which was transformed into categories representing inadequate health literacy (0-34), marginal health literacy (35-42) and adequate health literacy (43-64), but we categorized health literacy as a dichotomous variable between inadequate health literacy (0-34) and adequate health literacy (35-64) by merging the scores of marginal and adequate health literacy [24, 44, 45], only for data analysis and to have very clear and conclusive evidence of the impact of $\mathrm{HL}$, literate (adequate $\mathrm{HL}$ ) and illiterate (inadequate $\mathrm{HL}$ ), on both physical and mental HRQoL component scores.

Medication adherence (MA) was also considered as an independent variable for HRQoL but was considered dependent variable in case of HL. MA was assessed using, Morisky Green Levine Scale (MGLS), which is a four-item tool with a suggestive level of MA from high, medium to low with a score ranging from 0 to 4 . Morisky Green Levine Scale (MGLS) consisted of 4 items with "yes" and "no" options. The question answered as yes will be scored " 1 " while the question answered no will be scored "0". However, MGLS has also been described on the basis of dichotomous definition of adherence, with $1+$ points indicating some level of medication nonadherence and 0 indicating perfect level of adherence. Thus, a score between 3-4 (low adherence) and 1-2 (medium adherence) will indicate non-adherence and a score of 0 (high adherence) will indicate MA/perfect adherence [46, 47].

\section{Dependent or outcome variable}

Health related quality of life (HRQoL) was measured using 12-Item Short Form Survey (SF-12) version 1.0, a non-proprietary version, and the scores were calculated as described previously in original publication [48]. SF-12 (a multipurpose short form) is a shorter alternative to SF-36 and is becoming an instrument of choice to be used in measuring health of both general and specific populations. SF-12 can be administered in two minutes or less and can be either self-administered or through personal interviews [49], SF-12 questionnaire was summarized in to two measures; physical and mental components, which were scored using norm-based methods. Both the PCS-12 (Physical component summary scale12) and MCS-12 (Mental component summary scale-12) were transformed to contain a mean of 50 and a standard deviation of 10 and all the results were interpreted in accordance with the mean [48].

\section{Covariates}

Covariates, such as sociodemographic and clinical variables were selected based on the literature reviews $[7,8$, $24,50]$. Sociodemographic variables included age (greater or less than 65 years), gender (male or female), marital status (married or unmarried), residence area (rural or 
urban), education status (no schooling to primary; secondary to higher education), economic status (upper or lower), working status (employed full or part time; unemployed) and clinical variables, such as type of ischemic condition (angina or myocardial infarction), history of revascularization (yes or no), time since first ischemic condition (up to 5 years; more than 5 years), number of other CVD conditions (heart failure, stroke, arrhythmias, cardiomyopathy, heart block, and left ventricular dysfunction) were determined and then converted into ordinal variables $(0$ and $1+\mathrm{CVD}$ conditions) and risk factors (Being overweight, diagnosed with hypertension, dyslipidemia, chronic kidney disease and diabetes mellitus) were also determined and later turned into ordinal variables ( $0-1,2$ and $3+$ risk factors). Association of $\mathrm{HL}$ and MA was also estimated with co-variates affecting HRQoL.

\section{Study size}

Sample size for the study was determined using Cochran formula [51], taking into account the prevalence of IHD in Pakistan, i-e., $6.25 \%$ as of 2009 [52] with 5\% precision, $95 \%$ confidence interval and infinite population size. The sample size was found to be 91 as per the calculation, yet, 251 eligible IHD patients were enrolled in the study by random selection to compensate the drop outs due to missing data.

\section{Statistical methods}

Chi-square test was used to determine frequency distribution of categorical variables with respect to health literacy. Confidence intervals of $95 \%$ were also determined. The differences in the mean scores of physical and mental components of Health-related quality of life among sociodemographic and clinical variables was determined by using independent $\mathrm{t}$-test.

Descriptive statistics was used to determine the mean of physical and mental components of quality of life. Binary and multinomial regression were used to measure the associations. Linear regression model was used to evaluate $\beta$-regression coefficients, crude and adjusted, to determine the possible predictors of HRQoL. P-value of $<0.05$ was used as an indicator of statistical significance. Data were analyzed using IBM SPSS Statistics Version 20 .

\section{Results}

\section{Demographics and clinical characteristics of IHD patients} according to health literacy level

Patient's demographics and clinical characteristics as per health literacy [52] levels, adequate $\mathrm{HL}$ $\left(\mathbf{H L}_{\mathrm{AD}}\right)$ and inadequate $\mathrm{HL}\left(\mathbf{H L}_{\mathrm{IAD}}\right)$, are summarized in Table 1. Data revealed that majority of the patients $(>70 \%)$ were $<65$ years of age with no difference in the frequency distribution of adequate and inadequate HL levels, though scores were markedly different in both age groups based on which HL levels were defined, adequate and inadequate (scores; $<65: \mathbf{H L}_{\mathrm{IAD}} ; 11.98 \pm 10.44, \mathbf{H L}_{\mathrm{AD}} ; 48.71 \pm 8.89$, $\left.>65: \mathrm{HL}_{\mathrm{IAD}} ; 11.48 \pm 10.74, \mathrm{HL}_{\mathrm{AD}} ; 49.18 \pm 9.61\right)$. Compared to females, males $\left(\mathrm{HL}_{\mathrm{AD}} ; 78.7 \%, \mathbf{H L}_{\mathrm{IAD}} ; 55.7 \%\right.$, $p=0.001)$ had adequate HL. Patients from middle class $(50.7 \%)$ families (scores: $H_{L_{I A D}}$ 19.72 \pm 10.69 , $\left.\mathrm{HL}_{\mathrm{AD}} ; 50.47 \pm 8.89\right)$ and those having secondaryhigher secondary education $(74.7 \%, p=0.0001$,

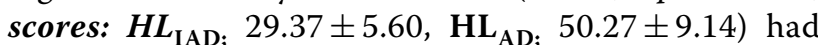
adequate $\mathrm{HL}$, while patients belonging to lower class (83.5\%, $\mathrm{p}=0.001$, scores: $\boldsymbol{H} \mathbf{L}_{\mathrm{IAD}} ; 10.2 \pm 9.57, \mathbf{H L}_{\mathrm{AD}}$; $46.32 \pm 8.41)$ families had inadequate HL. Moreover, the frequency distribution regarding employment status $(p=0.005)$, majority unemployed (scores: $H_{\text {IAD; }}$ $\left.11.37 \pm 10.22, \mathbf{H L}_{\mathrm{AD}} ; 48.15 \pm 9.08\right)$, and history of vascularization ( $p=0.0001$, scores: $H L_{\text {IAD; }} 14.05 \pm 12.02$, $\left.\mathbf{H L}_{\mathrm{AD}} ; 49.61 \pm 8.38\right)$ differed significantly with $\mathrm{HL}$ level. However, HL levels were not significantly different with regards to area of residence $(p=0.682)$, types of ischemic condition $(p=0.619)$, no. of other CVD conditions $(p=0.891)$, no. of clinical risk factors $(p=0.724)$ and MA $(p=0.229)$ (Table 1$)$.

\section{Physical and mental component summary of HRQoL in IHD patients as per $\mathrm{HL}$ level}

The physical (PCS-12) and mental (MCS-12) component summaries of HRQoL in IHD patients as per health literacy levels are summarized in Table 2. Data suggested that PCS-12 scores were significantly different with regards to HL for age $<65(p=0.001)$ and $>65(p=0.001)$ years, gender; male $(p=0.001)$ and female $(p=0.008)$, area of residence; urban $(p=0.001)$ and rural $(p=0.001)$, primary education $(p=0.045)$, socioeconomic status; upper $(p=0.026)$ and lower $(p=0.001)$, employment status; employed $(p=0.001)$ and un-employed $(p=0.001)$, history of re-vascularization, yes $(p=0.001)$ and no $(p=0.001)$, other CVD conditions; $0(p=0.001)$ and $\geq 1$ $(p=0.008)$, clinical CVD risk factors $(p<0.005)$ and MA; adequate $(p=0.023)$ (Table 2$)$.

Likewise, the MCS-12 scores were significantly different with regards to HL for area of residence; urban $(p=0.001)$ and rural $(p=0.014)$, no or primary education $(p=0.01)$, socio-economic status; lower $(p=0.001)$ and upper $(p=0.026)$, and MA; adherent $(p=0.0053)$. While contrary to PCS-12 scores, MCS-12 scores exhibited differences with regards to HL only for patients $<65$ years $(p=0.001)$ of age, male gender $(p=0.002)$, un-employed status $(p=0.016)$, no history of re-vascularization 
Table 1 Demographic clinical characteristics of Ischemic heart disease (IHD) patient according to health literacy

\begin{tabular}{|c|c|c|c|c|c|}
\hline \multirow[t]{3}{*}{ Characteristics } & \multicolumn{4}{|c|}{ Health literacy, $n=251$} & \multirow[t]{3}{*}{$p$ values } \\
\hline & \multicolumn{2}{|c|}{ Inadequate, $n=176$} & \multicolumn{2}{|c|}{ Adequate, $n=75$} & \\
\hline & Frequency (\%) & Average score $\pm S D$ & Frequency (\%) & Average score $\pm S D$ & \\
\hline \multicolumn{6}{|l|}{ Age (years) } \\
\hline$<65$ & $128(72.7)$ & $11.98 \pm 10.44$ & $58(77.3)$ & $48.71 \pm 8.89$ & \multirow[t]{2}{*}{0.446} \\
\hline$>65$ & $48(27.3)$ & $11.48 \pm 10.74$ & $17(22.7)$ & $49.18 \pm 9.61$ & \\
\hline \multicolumn{6}{|l|}{ Gender } \\
\hline Male & $98(55.7)$ & $14.90 \pm 11.32$ & $59(78.7)$ & $49.22 \pm 8.77$ & \multirow[t]{2}{*}{$0.001^{* *}$} \\
\hline Female & $78(44.3)$ & $8.01 \pm 7.88$ & $16(21.3)$ & $47.31 \pm 9.93$ & \\
\hline \multicolumn{6}{|l|}{ Residence area } \\
\hline Urban & $154(87.5)$ & $12.39 \pm 10.64$ & $67(89.3)$ & $49.27 \pm 9.07$ & \multirow[t]{2}{*}{0.682} \\
\hline Rural & $22(12.5)$ & $8.05 \pm 8.66$ & $8(10.7)$ & $45.00 \pm 7.82$ & \\
\hline \multicolumn{6}{|l|}{ Educational status } \\
\hline No_-primary education & $160(90.9)$ & $10.09 \pm 9.178$ & $19(25.3)$ & $44.53 \pm 7.14$ & \multirow[t]{2}{*}{$0.0001^{* *}$} \\
\hline Secondary_-higher education & $16(19.1)$ & $29.37 \pm 5.60$ & $56(74.7)$ & $50.27 \pm 9.14$ & \\
\hline \multicolumn{6}{|l|}{ Social status } \\
\hline Upper & $4(2.3)$ & $23 \pm 15.47$ & $6(8)$ & $51.17 \pm 10.98$ & \multirow[t]{3}{*}{$0.0001^{* *}$} \\
\hline Middle & $25(14.2)$ & $19.72 \pm 10.69$ & $38(50.7)$ & $50.47 \pm 8.89$ & \\
\hline Lower & $147(83.5)$ & $10.2 \pm 9.57$ & $31(41.3)$ & $46.32 \pm 8.41$ & \\
\hline \multicolumn{6}{|l|}{ Employment status } \\
\hline Employed full or part time & $34(19.3)$ & $15.38 \pm 11.00$ & $27(36.0)$ & $50.00 \pm 8.88$ & \multirow[t]{2}{*}{$0.005^{*}$} \\
\hline Unemployed & $142(80.7)$ & $11.00 \pm 10.22$ & $48(64.0)$ & $48.15 \pm 9.08$ & \\
\hline \multicolumn{6}{|l|}{ Type of ischemic condition } \\
\hline Angina & $35(19.9)$ & $12.34 \pm 10.11$ & $17(22.7)$ & $45.00 \pm 8.53$ & \multirow[t]{2}{*}{0.619} \\
\hline Myocardial Infarction & $141(80.1)$ & $11.72 \pm 10.62$ & $58(77.3)$ & $49.93 \pm 8.88$ & \\
\hline \multicolumn{6}{|l|}{ History of revascularization } \\
\hline Yes & $43(24.4)$ & $14.05 \pm 12.02$ & $38(50.7)$ & $49.61 \pm 8.38$ & \multirow[t]{2}{*}{$0.0001^{* *}$} \\
\hline No & $133(75.6)$ & $11.14 \pm 9.90$ & $37(49.3)$ & $48.00 \pm 9.63$ & \\
\hline \multicolumn{6}{|c|}{ Time since first ischemic episodes (years) } \\
\hline$\leq 5$ & $135(76.7)$ & $12.30 \pm 10.6$ & $57(76.0)$ & $49.09 \pm 8.84$ & \multirow[t]{2}{*}{0.904} \\
\hline$>5$ & $41(23.3)$ & $10.34 \pm 10.10$ & $18(24.0)$ & $47.94 \pm 9.67$ & \\
\hline \multicolumn{6}{|l|}{ Number of other CVD conditions } \\
\hline 0 & $161(91.5)$ & $11.98 \pm 10.59$ & $69(92.0)$ & $49.45 \pm 9.04$ & \multirow[t]{2}{*}{0.891} \\
\hline$\geq 1$ & $15(8.5)$ & $10.40 \pm 9.58$ & $6(8.0)$ & $41.50 \pm 4.04$ & \\
\hline \multicolumn{6}{|l|}{ Number of CVD risk factors } \\
\hline $0-1$ & $58(33.0)$ & $15.76 \pm 11.61$ & $24(32.0)$ & $48.75 \pm 8.86$ & \\
\hline 2 & $57(32.4)$ & $9.65 \pm 9.43$ & $28(37.3)$ & $48.14 \pm 8.53$ & 0.724 \\
\hline$\geq 3$ & $61(34.7)$ & $10.18 \pm 9.37$ & $23(30.7)$ & $49.70 \pm 9.97$ & \\
\hline Medication adherence & & & & & \\
\hline Adherent & $136(77.3)$ & $12.16 \pm 10.14$ & $63(84)$ & $49.06 \pm 8.91$ & 0.229 \\
\hline Non-adherent & $40(22.7)$ & $10.78 \pm 11.68$ & $12(16)$ & $47.50 \pm 9.72$ & \\
\hline
\end{tabular}

$p$ values were estimated based on differences in frequency distribution

$\mathrm{CVD}=$ cardiovascular disease. Health Literacy $(\mathrm{HL}) \mathrm{Scores}$; Inadequate =0-34, Adequate =35-64, SD; standard deviation

${ }^{*} p \leq 0.05-0.002,{ }^{* *} \leq 0.001-0.0001$

( $p=0.006)$, no other CVD condition $(p=0.001)$ and clinical CVD risk factors of $2(p=0.001)$ or $>3(p=0.002)$ (Table 2).
Association of health literacy with patient's demographics, clinical characteristics and MA

Data regarding the association of HL with demographics, clinical characteristics and MA in IHD patients 
Table 2 Physical and Mental component summary of HRQoL in IHD Patients according to Health Literacy Levels

\begin{tabular}{|c|c|c|c|c|c|c|}
\hline \multirow[t]{4}{*}{ Variables } & \multicolumn{6}{|c|}{ Health related quality of life (HRQoL) components } \\
\hline & \multicolumn{3}{|c|}{ PCS-12 (mean \pm SD) } & \multicolumn{3}{|c|}{ MCS-12 (mean \pm SD) } \\
\hline & \multicolumn{6}{|l|}{ Health literacy } \\
\hline & Inadequate & Adequate & $p$ value & Inadequate & Adequate & $p$ value \\
\hline \multicolumn{7}{|l|}{ Age } \\
\hline$<65$ years & $26.53 \pm 8.64$ & $37.62 \pm 12.54$ & $0.001^{* *}$ & $39.47 \pm 14.99$ & $49.06 \pm 11.97$ & $0.001^{* *}$ \\
\hline$>65$ years & $27.46 \pm 7.90$ & $39.83 \pm 10.47$ & $0.001^{* *}$ & $42.17 \pm 13.75$ & $47.00 \pm 10.37$ & 0.26 \\
\hline \multicolumn{7}{|l|}{ Gender } \\
\hline Male & $28.46 \pm 9.76$ & $37.62 \pm 11.97$ & $0.001^{* *}$ & $42.30 \pm 12.67$ & $49.32 \pm 11.25$ & $0.002^{*}$ \\
\hline Female & $24.68 \pm 5.78$ & $40.92 \pm 12.65$ & $0.008^{*}$ & $37.57 \pm 16.57$ & $44.61 \pm 12.99$ & 0.248 \\
\hline \multicolumn{7}{|l|}{ Residence area } \\
\hline Urban & $27.02 \pm 8.49$ & $37.80 \pm 12.50$ & $0.001^{* *}$ & $41.16 \pm 14.97$ & $48.51 \pm 11.85$ & $0.001^{* *}$ \\
\hline Rural & $25.16 \pm 7.97$ & $41.26 \pm 5.90$ & $0.001^{* *}$ & $33.52 \pm 10.39$ & $49.07 \pm 9.26$ & $0.014^{*}$ \\
\hline Educational status & & $36.68 \pm 13.48$ & $0.045^{*}$ & $39.79 \pm 15.03$ & $51.19 \pm 10.20$ & $0.01^{*}$ \\
\hline No_-primary education & $25.97 \pm 8.28$ & $38.47 \pm 11.83$ & 0.263 & $44.34 \pm 9.99$ & $47.99 \pm 11.85$ & 0.281 \\
\hline Secondary_-higher education & $35.00 \pm 4.84$ & & & & & \\
\hline \multicolumn{7}{|l|}{ Socioeconomic status } \\
\hline Upper & $30.13 \pm 8.27$ & $45.87 \pm 6.59$ & $0.026^{*}$ & $42.83 \pm 0.52$ & $49.75 \pm 9.27$ & $0.026^{*}$ \\
\hline Middle & $31.05 \pm 9.97$ & $37.07 \pm 11.91$ & 0.055 & $45.54 \pm 11.99$ & $47.74 \pm 12.64$ & 0.523 \\
\hline Lower & $25.97 \pm 7.96$ & $38.05 \pm 12.87$ & $0.001^{* *}$ & $39.22 \pm 15.12$ & $49.49 \pm 10.73$ & $0.001^{* *}$ \\
\hline \multicolumn{7}{|l|}{ Employment status } \\
\hline Employed & $30.79 \pm 10.22$ & $41.10 \pm 12.56$ & $0.001^{* *}$ & $45.36 \pm 14.09$ & $50.71 \pm 11.73$ & 0.111 \\
\hline Un-employed & $25.83 \pm 7.68$ & $33.47 \pm 9.97$ & $0.001^{* *}$ & $38.97 \pm 14.59$ & $33.24 \pm 9.98$ & $0.016^{*}$ \\
\hline \multicolumn{7}{|l|}{ Type of Ischemic condition } \\
\hline Angina & $28.67 \pm 10.50$ & $38.24 \pm 12.86$ & 0.081 & $40.49 \pm 13.17$ & $48.47 \pm 10.93$ & 0.120 \\
\hline Myocardial infarction & $26.32 \pm 7.81$ & $38.13 \pm 12.01$ & $0.001^{* *}$ & $40.13 \pm 15.07$ & $48.59 \pm 11.78$ & $0.001^{* *}$ \\
\hline \multicolumn{7}{|l|}{ History of revascularization } \\
\hline Yes & $27.87 \pm 8.09$ & $37.21 \pm 12.45$ & $0.001^{* *}$ & $45.83 \pm 12.81$ & $49.61 \pm 11.09$ & 0.200 \\
\hline No & $26.44 \pm 8.54$ & $39.44 \pm 11.56$ & $0.001^{* *}$ & $38.38 \pm 14.82$ & $47.13 \pm 12.26$ & $0.006^{*}$ \\
\hline \multicolumn{7}{|c|}{ Time since first ischemic episode (years) } \\
\hline$\leq 5$ & $27.42 \pm 9.34$ & $37.66 \pm 13.15$ & $0.001^{* *}$ & $41.42 \pm 15.06$ & $48.66 \pm 11.41$ & $0.002^{*}$ \\
\hline$>5$ & $25.5 \pm 6.38$ & $39.87 \pm 6.76$ & $0.001^{* *}$ & $36.89 \pm 12.52$ & $48.24 \pm 12.55$ & $0.017^{*}$ \\
\hline \multicolumn{7}{|l|}{ Other CVD conditions } \\
\hline 0 & $26.88 \pm 8.65$ & $38.13 \pm 12.37$ & $0.001^{* *}$ & $40.73 \pm 12.86$ & $48.91 \pm 11.80$ & $0.001^{* *}$ \\
\hline$\geq 1$ & $20.01 \pm 5.72$ & $38.43 \pm 3.77$ & $0.008^{*}$ & $35.64 \pm 28.38$ & $43.25 \pm 3.77$ & 0.658 \\
\hline \multicolumn{7}{|l|}{ Clinical CVD risk factors } \\
\hline $0-1$ & $28.47 \pm 8.86$ & $39.99 \pm 13.44$ & $0.004^{*}$ & $42.45 \pm 12.36$ & $43.57 \pm 11.65$ & 0.747 \\
\hline 2 & $26.52 \pm 9.08$ & $37.43 \pm 10.88$ & $0.001^{* *}$ & $40.49 \pm 17.35$ & $52.00 \pm 10.47$ & $0.001^{* *}$ \\
\hline$\geq 3$ & $25.43 \pm 7.14$ & $37.08 \pm 12.39$ & $0.003^{*}$ & $37.79 \pm 13.84$ & $49.56 \pm 11.57$ & $0.002^{*}$ \\
\hline \multicolumn{7}{|l|}{ Medication adherence } \\
\hline Adherent & $25.99 \pm 10.5$ & $34.91 \pm 11.31$ & $0.0235^{*}$ & $42.29 \pm 14.88$ & $51.3 \pm 10.12$ & $0.0053^{*}$ \\
\hline Non-adherent & - & $27.36 \pm 8.71$ & - & - & $38.12 \pm 11.69$ & - \\
\hline
\end{tabular}

$S D$ standard deviation, $C V D$ ardiovascular disease ${ }^{*} p \leq 0.05-0.002,{ }^{* *} \leq 0.001-0.0001$

are summarized in Table 3. As shown in Table 3., results of binary regression suggested that adequate literacy was significantly associated with secondary to higher education (OR; 19.3, $p=0.0001$ ), no history of re-vascularization (OR; $0.28, p=0.0001)$ and adequate MA (OR; 4.3, $p=0.014$ ). Besides, HL demonstrated no significant association with rest of the variables; demographic and clinical (Table 3). 
Table 3 Association of Health literacy with demographics, clinical characteristics and medication adherence in IHD patients

\begin{tabular}{|c|c|c|c|c|}
\hline \multirow[t]{2}{*}{ Characteristics } & \multicolumn{2}{|l|}{ Health literacy } & \multirow{2}{*}{$\begin{array}{l}\text { Binary logistic } \\
\text { OR (Cl) }\end{array}$} & \multirow[t]{2}{*}{$p$ values } \\
\hline & Adequate, $n=75$ & Inadequate, $n=176$ & & \\
\hline \multicolumn{5}{|l|}{ Age } \\
\hline$<65$ (reference) & 58 & 128 & $0.8(0.33-2.1)$ & 0.708 \\
\hline$>65$ & 17 & 48 & & \\
\hline \multicolumn{5}{|l|}{ Gender } \\
\hline Male & 59 & 98 & $1.3(0.54-2.9)$ & 0.59 \\
\hline Female (reference) & 16 & 78 & & \\
\hline \multicolumn{5}{|l|}{ Area of residence } \\
\hline Urban (reference) & 67 & 154 & $1.18(0.27-4.1)$ & 0.962 \\
\hline Rural & 8 & 22 & & \\
\hline \multicolumn{5}{|l|}{ Education } \\
\hline No_-primary (reference) & 19 & 160 & $19.3(8.3-45.1)$ & $0.0001^{* *}$ \\
\hline Secondary-higher & 56 & 16 & & \\
\hline \multicolumn{5}{|l|}{ Employment status } \\
\hline Employed & 27 & 34 & $1.94(0.82-4.6)$ & 0.132 \\
\hline Unemployed (reference) & 48 & 142 & & \\
\hline \multicolumn{5}{|l|}{ Socioeconomic status } \\
\hline Lower (reference) & 8 & 4 & & \\
\hline Middle & 38 & 25 & $2(0.35-11.8)$ & 0.43 \\
\hline Upper & 31 & 147 & $2.1(0.83-5.3)$ & 0.113 \\
\hline \multicolumn{5}{|l|}{ Time since first episode (years) } \\
\hline$\leq 5$ (reference) & 57 & 135 & $1.2(0.58-2.3)$ & 0.69 \\
\hline$>5$ & 18 & 41 & & \\
\hline \multicolumn{5}{|l|}{ History of re-vascularization } \\
\hline Yes (reference) & 38 & 43 & $0.28(0.15-0.51)$ & $0.0001^{* *}$ \\
\hline No & 37 & 133 & & \\
\hline \multicolumn{5}{|l|}{ Type of ischemic condition } \\
\hline Angina & 17 & 35 & $1.61(0.78-3.3)$ & 0.198 \\
\hline Myocardial infarction (reference) & 58 & 141 & & \\
\hline \multicolumn{5}{|l|}{ No. of CVD risk factors } \\
\hline 0-1 (reference) & 24 & 58 & $2.1(0.81-5.78)$ & 0.101 \\
\hline 2 & 28 & 57 & $1.3(0.48-3.6)$ & 0.534 \\
\hline$\geq 3$ & 23 & 61 & & \\
\hline \multicolumn{5}{|l|}{ Medication adherence } \\
\hline Adherent & 63 & 136 & $4.3(1.34-13.7)$ & $0.014^{*}$ \\
\hline Non-adherent (reference) & 12 & 40 & & \\
\hline
\end{tabular}

OR odds ratio, $C V D$ cardiovascular disease, $\mathrm{Cl}$ confidence interval

${ }^{*} p \leq 0.05-0.002 ;{ }^{* *} \leq 0.001-0.0001$

\section{Association of HRQoL, PCS-12 and MCS-12, with patient's demographics, clinical characteristics and MA}

Binary regression data presented in Table 4, revealed that IHD patients who were employed were more likely to exhibit improved PCS-12 scores (OR; 6.4, $p=0.004$ ), while no significant association was observed between PCS-12 and rest of the variables, nevertheless, IHD patients with adequate MA (OR; 3.2, $p=0.225)$ were more likely to demonstrate improved PCS-12 scores.
Moreover, HRQoL, mental component summary (MCS-12) association data suggested that IHD patients $<65$ years of age (OR; $2.2, p=0.034$ ), employment status (OR; $3, p=0.002$ ) and adequate MA (OR; $4, p=0.004$ ) were more likely to demonstrate improved MCS scores. While, no significant associations were observed for rest of the variables, nonetheless, the secondary to higher education (OR; $1.6, p=0.208)$ and no 
Table 4 Association of HRQoL, physical component summary (PCS-12) and mental component summary (MCS-12), with demographics, clinical characteristics and medication adherence in IHD patients

\begin{tabular}{|c|c|c|c|c|c|c|c|c|}
\hline \multirow[t]{2}{*}{ Characteristics } & \multicolumn{3}{|c|}{ PCS- $12, n=251$} & \multirow[t]{2}{*}{$p$ values } & \multicolumn{3}{|c|}{ MCS-12, $n=251$} & \multirow[t]{2}{*}{$p$ values } \\
\hline & $\begin{array}{l}\text { Improving } \\
(\geq 50), n=19\end{array}$ & $\begin{array}{l}\text { Not improving } \\
(<50) n=232\end{array}$ & $\begin{array}{l}\text { Binary logistic } \\
\text { OR (Cl) }\end{array}$ & & $\begin{array}{l}\text { Improving } \\
(\geq 50) \\
n=82\end{array}$ & $\begin{array}{l}\text { Not improving } \\
(<50) n=169\end{array}$ & $\begin{array}{l}\text { Binary logistic } \\
\text { OR (Cl) }\end{array}$ & \\
\hline Age & & & $1.1(0.34-18.4)$ & 0.91 & & & $2.2(1.1-4.94)$ & $0.034^{*}$ \\
\hline$<65$ & 16 & 170 & & & 57 & 129 & & \\
\hline$>65$ & 3 & 62 & & & 25 & 40 & & \\
\hline Gender & & & $1.6(0.37-6.7)$ & 0.544 & & & $1.7(0.87-3.3)$ & 0.120 \\
\hline Male & 16 & 141 & & & 61 & 96 & & \\
\hline Female & 3 & 91 & & & 21 & 73 & & \\
\hline \multicolumn{9}{|l|}{ Education } \\
\hline No_primary & 8 & 171 & & & 51 & 128 & & \\
\hline Secondary_higher & 11 & 61 & $3.5(0.93-13.1)$ & 0.063 & 31 & 41 & $1.6(0.75-3.7)$ & 0.208 \\
\hline \multicolumn{9}{|l|}{ Employment status } \\
\hline Employed & 12 & 49 & $6.4(1.8-22.5)$ & $0.004^{*}$ & 32 & 29 & $3(1.5-6.2)$ & $0.002^{*}$ \\
\hline Unemployed & 7 & 183 & & & 50 & 140 & & \\
\hline Time since first episode (years) & & & & & & & $0.59(0.28-1.3)$ & 0.184 \\
\hline$\leq 5$ & 18 & 174 & $0.14(0.016-1.25)$ & 0.079 & 68 & 124 & & \\
\hline$>5$ & 1 & 58 & & & 14 & 45 & & \\
\hline \multicolumn{9}{|l|}{ History of re-vascularization } \\
\hline Yes & 7 & 74 & $0.69(0.19-2.5)$ & 0.567 & 36 & 45 & $1.72(0.91-3.3)$ & 0.098 \\
\hline No & 12 & 158 & & & 46 & 124 & & \\
\hline Type of ischemic condition & & & $1.8(0.46-7.1)$ & 0.402 & & & $0.61(0.27-1.4)$ & 0.221 \\
\hline Angina & 5 & 47 & & & 18 & 34 & & \\
\hline Myocardial infarction & 14 & 185 & & & 64 & 135 & & \\
\hline \multicolumn{9}{|l|}{ No. of CVD risk factors } \\
\hline $0-1$ & 9 & 73 & $1.4(0.38-5.2)$ & 0.607 & 26 & 56 & $0.83(0.38-1.8)$ & 0.639 \\
\hline 2 & 5 & 80 & $0.77(0.18-3.24)$ & 0.717 & 32 & 53 & $1.6(0.73-3.3)$ & 0.256 \\
\hline$\geq 3$ & 5 & 79 & & & 24 & 60 & & \\
\hline Medication adherence & & & $3.2(0.49-20.1)$ & 0.225 & & & $4(1.6-10.3)$ & $0.004^{*}$ \\
\hline Adherent & 17 & 182 & & & 74 & 125 & & \\
\hline Non-adherent & 2 & 50 & & & 8 & 44 & & \\
\hline
\end{tabular}

$O R$ odds ratio, $C V D$ cardiovascular disease, $\mathrm{Cl}$ confidence interval

${ }^{*} p \leq 0.05-0.002,{ }^{* *} \leq 0.001-0.0001$

history of re-vascularization (OR; $1.72, p=0.098)$ were more likely to have improved MCS-12 scores (Table 4).

\section{Predictors of HRQoL}

Data regarding predictors of HRQoL, PCS-12 and MCS-12, in IHD patients are summarized in Table 5. In both un-adjusted and adjusted model, HL scores (un-adjusted: $\beta ; 0.513, p=0.0001$, adjusted: $\beta ; 0.383$, $p=0.0001$ ), literacy levels, adequate (un-adjusted: $\beta$; $-0.412, p=0.0001$, adjusted: $\beta ;-0.196, p=0.005)$ and inadequate (un-adjusted: $\beta$; $0.417, p=0.0001$, adjusted: $\beta$; $0.208, p=0.005)$ were significantly associated with physical (PCS-12) HRQoL, while the MA scores (adjusted: $\beta$; -0.133, $p=0.018$ ) and levels of MA, high (adjusted: $\beta$;
$0.124, p=0.027$ ) and low (adjusted: $\beta ; 0.134, p=0.018$ ) were significantly associated with PCS-12 only in the adjusted model (Table 5).

Furthermore, mental (MCS-12) HRQoL was significantly associated with HL scores (adjusted: $\beta ; 0.241$, $p=0.0001$ ) and level of HL, adequate (adjusted: $\beta ;-0.224$, $p=0.0001$ ) and inadequate (adjusted: $\beta ; 0.270, p=0.0001$ ) in un-adjusted model only. Contrary to physical HRQoL, MCS-12 was significantly associated with MA scores (unadjusted: $\beta ;-0.175, p=0.005$, adjusted: $\beta ;-0.161, p=0.009)$ and level of MA, high (un-adjusted: $\beta ;-0.147, p=0.019$, adjusted: $\beta ;-0.163, p=0.007$ ) and low (un-adjusted: $\beta$; $0.151, p=0.017$, adjusted: $\beta ; 0.136, p=0.028)$ in both unadjusted and adjusted models. Besides, only MCS- 12 
Table 5 Predictors of HRQOL in IHD patients

\begin{tabular}{|c|c|c|c|c|}
\hline \multirow[t]{3}{*}{ Characteristics } & \multicolumn{4}{|l|}{ PCS-12 } \\
\hline & \multicolumn{2}{|l|}{ Unadjusted } & \multicolumn{2}{|l|}{ Adjusted $^{\ddagger}$} \\
\hline & $\beta$-coefficients (Cl) & $p$ values & $\beta$-coefficients (Cl) & $p$ values \\
\hline Health literacy score & 0.513 (0.21 to 0.33$)$ & $0.0001^{* *}$ & 0.383 (0.12 to 0.29$)$ & $0.0001^{* *}$ \\
\hline Adequate & $-0.412(-13.6$ to -7.7$)$ & $0.0001^{* *}$ & $-0.196(-8.6$ to -1.5$)$ & $0.005^{*}$ \\
\hline Marginal & -0.08 ( -7.3 to 1.2$)$ & 0.163 & $-0.016(-4.5$ to 3.4$)$ & 0.783 \\
\hline Inadequate & 0.417 (6.9 to 11.9) & $0.0001^{* *}$ & $0.208(1.4$ to 7.9$)$ & $0.005^{*}$ \\
\hline Medication adherence score & -0.123 (- 1.8 to 0.008$)$ & 0.052 & $-0.133(-1.7$ to -0.17$)$ & $0.018^{*}$ \\
\hline High & $-0.095(-4.6$ to 0.59$)$ & 0.132 & $-0.124(-4.9$ to -0.29$)$ & $0.027^{*}$ \\
\hline Medium & $0.006(-2.6$ to 2.9$)$ & 0.924 & $0.020(-2$ to 2.9$)$ & 0.725 \\
\hline Low & $0.110(-0.35$ to 5.9$)$ & 0.081 & 0.134 (0.58 to 6.3 ) & $0.018^{*}$ \\
\hline Time since first episode (years) & $-0.042(-4.1$ to 2$)$ & 0.510 & $-0.052(-3.9$ to 1.4$)$ & 0.356 \\
\hline History of re-vascularization & $-0.115(-5.3$ to 0.195$)$ & 0.069 & $-0.008(-2.7$ to 2.3$)$ & 0.889 \\
\hline Type of ischemic condition & $-0.076(-5.1$ to 1.2$)$ & 0.230 & $-0.076(-4.9$ to 0.97$)$ & 0.190 \\
\hline No. of CVD risk factors & $-0.136(-3.3$ to -0.16$)$ & $0.031^{*}$ & -0.091 ( -2.6 to 0.252$)$ & 0.107 \\
\hline \multirow[t]{3}{*}{ Characteristics } & \multicolumn{4}{|l|}{ MCS- 12} \\
\hline & \multicolumn{2}{|l|}{ Unadjusted } & \multicolumn{2}{|l|}{ Adjusted $^{\ddagger}$} \\
\hline & $\beta$-coefficients (Cl) & $p$ values & $\beta$-coefficients (Cl) & $p$ values \\
\hline Health literacy score & 0.241 (0.086 to 0.261$)$ & $0.001^{* *}$ & $0.107(-0.053$ to 0.207$)$ & 0.242 \\
\hline Adequate & $-0.224(-12.24$ to -3.6$)$ & $0.0001^{* *}$ & $-0.108(-9.1$ to 1.53$)$ & 0.16 \\
\hline Marginal & $0.103(-1.8$ to 11.6$)$ & 0.151 & $0.049(-4.6$ to 9.1$)$ & 0.505 \\
\hline Inadequate & 0.270 (3.9 to 12.7) & $0.0001^{* *}$ & $0.147(-1.2$ to 10.3$)$ & 0.119 \\
\hline Medication adherence score & $-0.175(-2.9$ to -0.51$)$ & $0.005^{*}$ & $-0.161(-2.7$ to -0.4$)$ & $0.009^{*}$ \\
\hline High & $-0.147(-7.7$ to -0.68$)$ & $0.019^{*}$ & $-0.163(-8$ to -1.3$)$ & $0.007^{*}$ \\
\hline Medium & $0.027(-2.9$ to 4.6$)$ & 0.676 & $0.078(-1.8$ to 5.5$)$ & 0.329 \\
\hline Low & 0.151 (0.97 to 9.6) & $0.017^{*}$ & 0.136 (0.52 to 9) & $0.028^{*}$ \\
\hline Time since first episode (Yrs) & $-0.096(-7.4$ to 0.93$)$ & 0.127 & $-0.107(-7.6$ to 0.43$)$ & 0.080 \\
\hline History of re-vascularization & $-0.225(-10.5$ to -3.1$)$ & $0.0001^{* *}$ & $-0.162(-8.6$ to -1.2$)$ & $0.009^{*}$ \\
\hline Type of ischemic condition & $-0.009(-4.7$ to 4$)$ & 0.886 & $-0.046(-5.9$ to 2.7$)$ & 0.469 \\
\hline No. of CVD risk factors & $-0.070(-3.4$ to 0.95$)$ & 0.268 & $-0.033(-2.7$ to 1.5$)$ & 0.587 \\
\hline
\end{tabular}

CVD cardiovascular disease, $P C S$ physical component summary, MCS mental component summary

${ }^{*} p \leq 0.05-0.002,{ }^{* *} \leq 0.001-0.0001, \neq$ Adjusted for Co-variates; age, gender, marital status, education, area of residence, employment status

was significantly associated with history of re-vascularization (un-adjusted: $\beta ;-0.225, p=0.0001$, adjusted: $\beta$; $-0.162, p=0.009)$ in both unadjusted and adjusted models (Table 5).

No significant associations were observed for rest of the variables with either physical or mental HRQoL (Table 5).

\section{Discussion}

Numerous literature reports suggest that health literacy can impact the quality of life of CVD patients, yet most of these have been limited to the patients with heart failure $[35,53]$. The intricate relationship between $\mathrm{HL}$ and HRQoL is explained by insolvent adherence to life style changes and pharmacological treatments among patients with inadequate health literacy affecting
HRQoL $[28,35]$. Yet the association of HL and HRQoL with IHD patient's demographics, clinical characteristics and MA remain unclear. In the present study, the first from Pakistan, we observed that in IHD patients HL literacy was significantly different with regards to age, education, employment status, social class and history of re-vascularization. Likewise, based on HL, physical and mental health components of HRQoL were significantly different in IHD patients $<65$ years of age, no or primary education, upper and lower social class, un-employed, no history of re-vascularization, 2 or more clinical CVD risk factors and adequate MA. Additionally, HRQoL, PCS-12, was significantly associated with $\mathrm{HL}$, adequate and in-adequate levels, MA score, high and poor adherence levels, even when 
adjusted for co-variates. While, MCS-12 was significantly associated with HL score and HL levels in unadjusted model only, however, MA score, high and low adherence levels and history of re-vascularization demonstrated significant association with MCS-12 even when adjusted for co-variates.

During the past decades, besides traditional end points that may not depict the impact of interventions on patient's HRQoL, there has been growing concern and interest in assessing the patient's HRQoL, especially in patients with chronic diseases [54]. In this context, the chronic nature of IHD requires the patient to adequately understand the disease and therapy in order to actively participate in the management of his/ her health condition. We observed that only 30\% IHD patients exhibited adequate HL, out of which majority of them were males, had secondary/higher secondary education, hailing from the middle class, employed and were adherent to their medication. Albeit, no study was available from Pakistan on IHD patients for direct comparison, yet a study on elderly hypertensive patients of Islamabad, federal capital city, reported adequate HL in $37.4 \%$ subjects [55]. We have reported previously that HL was $33 \%$ among diabetic patients, more closer to the findings of the current study, with higher frequency of adequate HL in middle class, well-educated and employed subjects [14]. The differences in the HL levels reported in our studies and the one conducted in Islamabad on elderly hypertensive patients may be attributed to the differences in the $\mathrm{HL}$ assessment tools, more complex disease condition, i-e., IHD, and more educated working-class residents in Islamabad with white collar jobs compared to Lahore. Additionally, similar to our findings, studies from Taiwan [56], Serbia [57] and Spain [58] reported adequate HL of 36\%, 38.9\% and $21 \%$, respectively, among CVD patients.

Moreover, HL is greatly associated with MA [59], since inadequate HL leads to weak or almost no MA [60]. We observed that $47.4 \%$ of the IHD patients had adequate MA, which was significantly associated with HL. Two studies from federal capital city, Islamabad, though direct comparison cannot be made, reported adequate MA of $38.9 \%$ [55] and 38.3\% [61] in hypertensive patients, while one study demonstrated significant association of HL with adequate MA [55] - corroborating our findings, yet in different disease condition. It has been assumed that the presence of co-morbid conditions, quite common in IHD patients, modulate the attitude of such patients towards a stricter medication-taking behavior [62], which could be one reason of the improved MA in IHD patients compared to hypertensive population of Islamabad.

Several lines of literature evidences suggest considerable association of HRQoL with HL $[35,53]$ and MA in patients with chronic diseases [63]. Yet, only a few studies evaluated the impact of HL and MA on IHD patient's HRQoL, not a single from Pakistan. Our data suggested that both physical and mental component summary scores, segregated based on demographic and clinical parameters, were significantly higher in subjects with adequate HL-though inadequate HL exhibited stronger effect on physical component of HRQoL in IHD patients as reported previously for patients with chronic diseases [24, 64]. Besides, no significant associations were observed for both physical and mental components of HRQoL with patient characteristics except for employment status-suggesting that the patient's ability to go for a job and earn would have a positive impact on HRQoL, particularly relating to the emotional dimension of HRQoL. We further observed that MCS-12 was significantly associated with adequate MA, a finding that is in agreement with previously published reports that MA is significantly associated with mental component of HRQoL in patients with pulmonary tuberculosis [65] and older hypertensive patients [66]. Data further suggested that in IHD patients, health literacy was an independent predictor of HRQoL, physical and mental components, however, when adjusted for co-variates, HL still remained an independent predictor of physical dimension of HRQoL, corroborated by previous report in heart failure patients [57]. MA and HRQoL are important indicators of therapeutic success, while adherence to therapy drives changes in HRQoL [67]. We observed that MA was significantly associated with emotional dimension of HRQoL, un-adjusted and adjusted, but its association with physical dimension of HRQoL was observed only when adjusted for co-variates. These data clearly demonstrated that $\mathrm{HL}$ and MA are independent predictors of HRQoL, though with variables impact on physical and emotional dimensions of HRQoL.

\section{Study clinical implication and policy recommendations}

In the hierarchical cluster of chronic disease management, understanding about the disease and therapy, i.e., HL lies on the top, the effect of which trickles down to any linked actions on patient's side, such self-care and management in form of adherence to therapy and life style modifications having far reaching impact on patient's HRQoL. We and others have shown previously that in developing countries, including Pakistan, where majority have poor finances and limited access to education, HL is an unexplored entity, created and refined by the developed world-though people in Pakistan are conscientiously oblivious of the term HRQoL. Unlike, diabetes, IHD patients require more intricate self-care and management stratagems that implicitly and exclusively demand minimal disease and therapy related 
understanding to practice optimal self-care and management skills having significant impact on HRQoL. Therefore, much responsibility lies on health professionals in refining HL in IHD patients, which is not only associated to MA but in turn would improve HRQoL in IHD patients.

In this regard, integrative patient-centered approach by a team of skilled health professionals, doctors, pharmacists and nurses, can play pivotal role in improving HRQoL in IHD patients. As HRQoL cannot be extrapolated from routine clinical variables, therefore, in IHD patients, HRQoL should specifically be assessed in routine clinical examination to better treat the non-physical aspects of the disease having direct bearing on the management and the promotion of HRQoL in IHD patients. Besides, as observed in our study, HL is significantly associated with MA and HRQoL in IHD patients, by achieving marginal to adequate HL levels would immanently improve both the MA and HRQoL in these patients. Thus, clinical pharmacist services should be mandated to educate and counsel IHD patients to achieve these non-clinical, yet pivotal targets with upfront influence on HRQoL.

\section{Study limitations}

Our study has a few limitations. The cross-sectional design of the study did not permit to observer patients over a period of time and interpret causal relationship between the variables. Also, the small sample size $(n=251)$ would limit the generalizability of the study findings. Moreover, face to face interviews were conducted for data collection that may lead to reporting bias due to the influence of social desirability factor, which might compel the participants to give desirable answers related to their quality of life to the interviewer. Finally, direct comparison cannot be done due to non-availability of any similar study in IHD patients from Pakistan.

\section{Conclusions}

In conclusion, our results suggest that IHD patients having inadequate HL have significantly reduced physical and psychological dimensions of HRQoL, but with more significant impact on physical functioning. Besides, adherent IHD patients had adequate HL and improved physical and psychological dimensions of HRQoL. Data further suggested that HL and MA are independent predictors of HRQoL in IHD patients. Therefore, during every consultation with IHD patients, health professionals, doctors and pharmacists, should consider inadequate $\mathrm{HL}$ as a potential barrier to MA and provide effective care that has significant impact on health outcomes with subsequent effects on physical dimensions of HRQoL.

\section{Abbreviations}

HL: Health literacy; MA: Medication adherence; HRQoL: Health related quality of life; IHD: Ischemic heart disease; MGLS: Morisky Green Levine Scale; SF-12: 12-Item Short Form Survey; BR: Binary regression; MR: Multinomial regression; $\mathrm{HL}_{\mathrm{AD}}$ : Adequate health literacy; $\mathrm{HL}_{\mathrm{IAD}}$ : Inadequate health literacy; PCS-12:

Physical component summary-12; MCS-12: Mental component summary-12.

\section{Acknowledgements}

Authors are thankful to the hospital staff and the participants for their kind cooperation.

\section{Authors' contributions}

MS; collected and analyzed data and wrote the manuscript, HS; designed and supervised the study, analyzed data and wrote the manuscript, ZS; designed the study and analyzed the data, SY; collected and analyzed the data, FKH; analyzed data and provided resources, FR; provided resources, analyzed data and edited the manuscript, Ml; co-supervised the study and helped in data analysis, II; provided logistic support and helped in data collection. All authors read and approved the final manuscript.

Funding

No funds from any source, public or private, were obtained for this study.

Availability of data and materials

Can be obtained from corresponding author upon a reasonable request.

\section{Ethics approval}

The study received medical and ethical approval from Punjab University College of Pharmacy and Institutional review board of Mayo Hospital Lahore, Pakistan. All patients and their families were informed and agreed to the study.

\section{Consent for publication}

Not applicable.

\section{Competing interests}

Authors declared that no competing interests exist.

\section{Author details}

${ }^{1}$ Department of Pharmaceutics, University College of Pharmacy, Universality of the Punjab, Allama lqbal Campus, Lahore 54000, Pakistan. ${ }^{2}$ Department of Pharmacy, The University of Lahore, Lahore, Pakistan. ${ }^{3}$ Department of Pharmacy Practice, Faculty of Pharmacy, Bahauddin Zakariya University, Multan, Pakistan. ${ }^{4}$ Department of Pharmacology, Faculty of Pharmacy, Bahauddin Zakariya University, Multan, Pakistan.

Received: 15 September 2020 Accepted: 3 April 2021

Published online: 13 April 2021

\section{References}

1. World Health Organization. WHO maps: non-communicable disease trend in all countries. World Health Global Report, World Health Organization; 2011.

2. Balakumar P, Maung-U K, Jagadeesh G. Prevalence and prevention of cardiovascular disease and diabetes mellitus. Pharmacol Res. 2016;113:600-9.

3. Reddy KS, Yusuf S. Emerging epidemic of cardiovascular disease in developing countries. Circulation. 1998;97(6):596-601.

4. Turin TC, Shahana N, Wangchuk LZ, Specogna AV, Al Mamun M, Khan MA, Choudhury SR, Zaman MM, Rumana N. Burden of cardio-and cerebrovascular diseases and the conventional risk factors in South Asian population. Glob Heart. 2013;8(2):121-30.

5. Yusuf S, Rangarajan S, Teo K, Islam S, Li W, Liu L, Bo J, Lou Q, Lu F, Liu T. Cardiovascular risk and events in 17 low-, middle-, and high-income countries. N Engl J Med. 2014;371(9):818-27.

6. Hong E. Health-related quality of life and health condition of communitydwelling populations with cancer, stroke, and cardiovascular disease. J Phys Ther Sci. 2015;27(8):2521-4.

7. Ludt S, Wensing M, Szecsenyi J, Van Lieshout J, Rochon J, Freund T, Campbell SM, Ose D. Predictors of health-related quality of life in patients 
at risk for cardiovascular disease in European primary care. PLOS ONE. 2011;6(12):e29334.

8. Smith SG, O'Conor R, Curtis LM, Waite K, Deary IJ, Paasche-Orlow M, Wolf MS. Low health literacy predicts decline in physical function among older adults: findings from the LitCog cohort study. J Epidemiol Community Health. 2015;69(5):474-80

9. Marathe J, Ogden K, Woodroffe J. Health literacy, genetic literacy and outcomes in patients with genetic cardiac disease: a review of the literature. Heart Lung Circ. 2014;23:e12.

10. Evangelista LS, Rasmusson KD, Laramee AS, Barr J, Ammon SE, Dunbar S, Ziesche S, Patterson JH, Yancy CW. Health literacy and the patient with heart failure-implications for patient care and research: a consensus statement of the Heart Failure Society of America. J Cardiac Fail. 2010;16(1):9-16.

11. Lemay J, Waheedi M, Al-Sharqawi S, Bayoud T. Medication adherence in chronic illness: do beliefs about medications play a role? Patient Prefer Adherence. 2018;12:1687.

12. Gazmararian JA, Kripalani S, Miller MJ, Echt KV, Ren J, Rask K. Factors associated with medication refill adherence in cardiovascular-related diseases. J Gen Intern Med. 2006;21(12):1215-21.

13. Zolnierek KB, Dimatteo MR. Physician communication and patient adherence to treatment: a meta-analysis. Med Care. 2009;47(8):826-34.

14. Saeed H, Saleem Z, Naeem R, Shahzadi I, Islam M. Impact of health literacy on diabetes outcomes: a cross-sectional study from Lahore, Pakistan. Public Health. 2018;156:8-14.

15. Miller TA. Health literacy and adherence to medical treatment in chronic and acute illness: a meta-analysis. Patient Educ Couns. 2016;99(7):1079-86.

16. Lu M, Ma J, Lin Y, Zhang X, Shen Y, Xia H. Relationship between patient's health literacy and adherence to coronary heart disease secondary prevention measures. J Clin Nurs. 2019;28(15-16):2833-43.

17. Thakkar J, Kurup R, Laba TL, Santo K, Thiagalingam A, Rodgers A, Woodward M, Redfern J, Chow CK. Mobile telephone text messaging for medication adherence in chronic disease: a meta-analysis. JAMA Intern Med. 2016;176(3):340-9.

18. Wang C, Lang J, Xuan L, Li X, Zhang L. The effect of health literacy and self-management efficacy on the health-related quality of life of hypertensive patients in a western rural area of China: a cross-sectional study. Int J Equity Health. 2017;16(1):58.

19. Ho PM, Spertus JA, Masoudi FA, Reid KJ, Peterson ED, Magid DJ, Krumholz HM, Rumsfeld JS. Impact of medication therapy discontinuation on mortality after myocardial infarction. Arch Intern Med. 2006;166(17):1842-7.

20. Bansilal S, Castellano JM, Garrido E, Wei HG, Freeman A, Spettell C, Garcia-Alonso F, Lizano I, Arnold RJ, Rajda J, et al. Assessing the impact of medication adherence on long-term cardiovascular outcomes. J Am Coll Cardiol. 2016;68(8):789-801.

21. Bahall M, Legall G, Khan K. Quality of life among patients with cardiac disease: the impact of comorbid depression. Health Qual Life Outcomes. 2020;18(1):189.

22. Samiei Siboni F, Alimoradi Z, Atashi V, Alipour M, Khatooni M. Quality of life in different chronic diseases and its related factors. Int J Prev Med. 2019;10:65.

23. Park NH, Song MS, Shin SY, Jeong Jh, Lee HY. The effects of medication adherence and health literacy on health-related quality of life in older people with hypertension. Int J Older People Nurs. 2018;13(3):e12196.

24. González-Chica DA, Mnisi Z, Avery J, Duszynski K, Doust J, Tideman P, Murphy A, Burgess J, Beilby J, Stocks N. Effect of health literacy on quality of life amongst patients with ischaemic heart disease in Australian general practice. PLOS ONE. 2016;11(3):e0151079.

25. Hussain N, Said AS, Khan Z. Influence of health literacy on medication adherence among elderly females with type 2 diabetes in Pakistan. Int Q Community Health Educ. 2019;2019:0272684X19896724

26. Bitton A, Choudhry NK, Matlin OS, Swanton K, Shrank WH. The impact of medication adherence on coronary artery disease costs and outcomes: a systematic review. Am J Med. 2013;126(4):357.e357-357.e327.

27. Uchmanowicz B, Chudiak A, Mazur G. The influence of quality of life on the level of adherence to therapeutic recommendations among elderly hypertensive patients. Patient Prefer Adherence. 2018;12:2593.

28. Westlake C, Sethares K, Davidson P. How can health literacy influence outcomes in heart failure patients? Mechanisms and interventions. Curr Heart Fail Rep. 2013;10(3):232-43.
29. Berkman DD, Pignone MP, Sheridan SL, Lohr KN, Lux L, Sutton SF, Swinson T, Bonito AJ. Literacy and health outcomes: summary. In: AHRQ evidence report summaries. AHRQ Publication Number 04-E007-1; 2004.

30. Howard DH, Gazmararian J, Parker RM. The impact of low health literacy on the medical costs of Medicare managed care enrollees. Am J Med. 2005;118(4):371-7.

31. Cutler RL, Fernandez-Llimos F, Frommer M, Benrimoj C, Garcia-Cardenas V. Economic impact of medication non-adherence by disease groups: a systematic review. BMJ Open. 2018;8(1):e016982.

32. Gast A, Mathes T. Medication adherence influencing factors - an (updated) overview of systematic reviews. Systems Control Found Appl. 2019:8(1):112.

33. Saeed A, Saeed H, Saleem Z, Fang Y, Babar Z-U-D. Evaluation of prices, availability and affordability of essential medicines in Lahore Division, Pakistan: a cross-sectional survey using WHO/HAl methodology. PLoS ONE. 2019;14(4):e0216122.

34. Chu SH, Lee WH, Yoo JS, Kim SS, Ko IS, Oh EG, Lee J, Choi M, Cheon JY, Shim CY. Factors affecting quality of life in Korean patients with chronic heart failure. Jpn J Nurs Sci. 2014;1 1 (1):54-64.

35. Macabasco-O'Connell A, DeWalt DA, Broucksou KA, Hawk V, Baker DW, Schillinger D, Ruo B, Bibbins-Domingo K, Holmes GM, Erman B. Relationship between literacy, knowledge, self-care behaviors, and heart failurerelated quality of life among patients with heart failure. J Gen Internal Med. 2011;26(9):979-86.

36. Al Sayah F, Majumdar SR, Williams B, Robertson S, Johnson JA. Health literacy and health outcomes in diabetes: a systematic review. J Gen Intern Med. 2013;28(3):444-52.

37. Gazmararian JA, Williams MV, Peel J, Baker DW. Health literacy and knowledge of chronic disease. Patient Educ Couns. 2003;51(3):267-75.

38. Sudore RL, Mehta KM, Simonsick EM, Harris TB, Newman AB, Satterfield S, Rosano C, Rooks RN, Rubin SM, Ayonayon HN. Limited literacy in older people and disparities in health and healthcare access. J Am Geriatr Soc. 2006;54(5):770-6.

39. Kamal AK, Muqeet A, Farhat K, Khalid W, Jamil A, Gowani A, Muhammad $A A$, Zaidi F, Khan D, Elahi T. Using a tailored health information technology-driven intervention to improve health literacy and medication adherence in a Pakistani population with vascular disease (Talking Rx) study protocol for a randomized controlled trial. Trials. 2016;17(1):1-13.

40. Chew LD, Bradley KA, Boyko EJ. Brief questions to identify patients with inadequate health literacy. Health. 2004;11:12.

41. Mayo Hospital Lahore, Pakistan. https://www.mayohospital.gop.pk/.

42. PAKISTAN BUREAU OF STATISTICS Government of Pakistan. http://www. pbs.gov.pk/content/provisional-summary-results-6th-population-andhousing-census-2017-0.

43. Fihn SD, Blankenship JC, Alexander KP, Bittl JA, Byrne JG, Fletcher BJ, Fonarow GC, Lange RA, Levine GN, Maddox TM. 2014 ACC/AHA/AATS/ PCNA/SCAI/STS focused update of the guideline for the diagnosis and management of patients with stable ischemic heart disease: a report of the American College of Cardiology/American Heart Association Task Force on Practice Guidelines, and the American Association for Thoracic Surgery, Preventive Cardiovascular Nurses Association, Society for Cardiovascular Angiography and Interventions, and Society of Thoracic Surgeons. Circulation. 2014;130(19):1749-67.

44. Tavakolikia N, Kheiltash A, Shojaeefar E, Montazeri A, Shariati M, Meysamie A. The most well-known health literacy questionnaires: a narrative review. Soc Determ Health. 2017;3(2):104-13.

45. Xia J, Wu P, Deng Q, Yan R, Yang R, Lv B, Wang J, Yu J. Relationship between health literacy and quality of life among cancer survivors in China: a cross-sectional study. BMJ Open. 2019;9(12):e028458.

46. Koschack J, Marx G, Schnakenberg J, Kochen MM, Himmel W. Comparison of two self-rating instruments for medication adherence assessment in hypertension revealed insufficient psychometric properties. J Clin Epidemiol. 2010;63(3):299-306.

47. Beyhaghi H, Reeve BB, Rodgers JE, Stearns SC. Psychometric properties of the Four-Item Morisky green levine medication adherence scale among atherosclerosis risk in communities (ARIC) study participants. Value in Health. 2016;19(8):996-1001.

48. Ware J, Kosinski M, Keller S. SF-12: how to score the SF-12 physical and mental health summary scales. 1998. 
49. Ware JE Jr, Kosinski M, Keller SD. A 12-Item Short-Form Health Survey: construction of scales and preliminary tests of reliability and validity. Med Care. 1996;34(3):220-33.

50. Kripalani S, Henderson LE, Chiu EY, Robertson R, Kolm P, Jacobson TA. Predictors of medication self-management skill in a low-literacy population. J Gen Intern Med. 2006;21(8):852-6.

51. Cochran WG. Sampling techniques. New York: Wiley; 1963.

52. Abbas S, Kitchlew A, Abbas S. Disease burden of Ischemic Heart Disease in Pakistan and its risk factors. Ann Pak Inst Med Sci. 2009:5(3):145-50.

53. Nesbitt T, Doctorvaladan S, Southard JA, Singh S, Fekete A, Marie K, Moser DK, Pelter MM, Robinson S, Wilson MD. Correlates of quality of life in rural patients with heart failure. Circul Heart Fail. 2014;7(6):882-7.

54. Al-Kaade S, Hauptman PJ. Health-related quality of life measurement in heart failure: challenges for the new millennium. J Cardiac Fail. 2001;7(2):194-201.

55. Saqlain M, Riaz A, Malik MN, Khan S, Ahmed A, Kamran S, Ali H. Medication adherence and its association with health literacy and performance in activities of daily livings among elderly hypertensive patients in Islamabad, Pakistan. Medicina. 2019;55(5):163.

56. Tung H-H, Lu T-M, Chen L-K, Liang S-Y, Wu S-F, Chu K-H. Health literacy impact on elderly patients with heart failure in Taiwan. J Clin Gerontol Geriatr. 2014;5(3):72-6.

57. Jovanić M, Zdravković M, Stanisavljević D, Jović Vraneš A. Exploring the importance of health literacy for the quality of life in patients with heart failure. Int J Environ Res Public Health. 2018;15(8):1761.

58. Santesmases-Masana R, Gonzalez-de Paz L, Real J, Borras-Santos A, SisoAlmirall A, Navarro-Rubio MD. Health literacy in patients with heart failure treated in primary care. Aten Primaria. 2016;49(1):28-34.

59. Berkman ND, Sheridan SL, Donahue KE, Halpern DJ, Crotty K. Low health literacy and health outcomes: an updated systematic review. Ann Intern Med. 2011:155(2):97-107.

60. Kripalani S, Gatti ME, Jacobson TA. Association of age, health literacy, and medication management strategies with cardiovascular medication adherence. Patient Educ Couns. 2010;81(2):177-81.
61. Mahmood S, Jalal Z, Hadi MA, Orooj H, Shah KU. Non-adherence to prescribed antihypertensives in primary, secondary and tertiary healthcare settings in Islamabad, Pakistan: a cross-sectional study. Patient Prefer Adherence. 2020;14:73.

62. Billups SJ, Malone DC, Carter BL. The relationship between drug therapy noncompliance and patient characteristics, health-related quality of life, and health care costs. Pharmacother J Hum Pharmacol Drug Ther 2000;20(8):941-9.

63. Krack G, Holle R, Kirchberger I, Kuch B, Amann U, Seidl H. Determinants of adherence and effects on health-related quality of life after myocardial infarction: a prospective cohort study. BMC Geriatr. 2018;18(1):136.

64. Wang C, Kane RL, Xu D, Meng Q. Health literacy as a moderator of healthrelated quality of life responses to chronic disease among Chinese rural women. BMC Womens Health. 2015;15(1):34.

65. Kastien-Hilka T, Rosenkranz B, Schwenkglenks M, Bennett BM, Sinanovic E. Association between health-related quality of life and medication adherence in pulmonary tuberculosis in South Africa. Front Pharmacol. 2017;8:919.

66. Peacock E, Joyce C, Craig LS, Lenane Z, Holt EW, Muntner P, Krousel-Wood M. Low medication adherence is associated with decline in health-related quality of life: results of a longitudinal analysis among older women and men with hypertension. J Hypert. 2020;39:153-61.

67. Côté I, Farris K, Feeny D. Is adherence to drug treatment correlated with health-related quality of life? Qual Life Res. 2003;12(6):621-33.

\section{Publisher's Note}

Springer Nature remains neutral with regard to jurisdictional claims in published maps and institutional affiliations.
Ready to submit your research? Choose BMC and benefit from:

- fast, convenient online submission

- thorough peer review by experienced researchers in your field

- rapid publication on acceptance

- support for research data, including large and complex data types

- gold Open Access which fosters wider collaboration and increased citations

- maximum visibility for your research: over $100 \mathrm{M}$ website views per year

At BMC, research is always in progress.

Learn more biomedcentral.com/submissions 